\title{
PARTICLE PRODUCTION THROUGH TWO-PHOTON PROCESSES IN RELATIVISTIC HEAVY-ION COLLIDERS
}

\author{
J.-S. Wu, C. Bottcher, M. R. Strayer, and C. M. Shakin
}

CONF-9105238--2

DE91 016221

\section{DISCLAIMER}

This report was prepared as an account of work sponsored by an agency of the United States Government. Neither the United States Government nor any agency thereof, nor any of their employees, makes any warranty, express or implied, or assumes any legal liability or responsibility for the accuracy, completeness, or usefulness of any information, apparatus, product, or process disclosed, or represents that its use would not infringe privately owned rights. Reference herein to any specific commercial prociact, process, or service by trade name, trademark, manufacturer, or otherwise does not necessarily constitute or imply its endorsement, recommendation. of favoring by the United States Government or any agency thereof. The views and opinions of authors expressed herein do not necessarily state or reflect those of the United States Government or any agency thereol.

\author{
to be published in
}

\section{Proceedings of Conference on Computational Quantum Physics \\ Nashville, Tennessee \\ May 22-25, 1991}

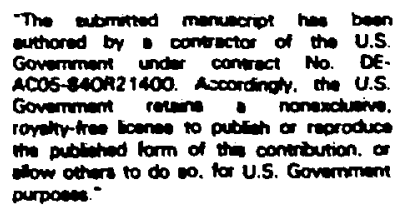




\title{
Particle Production through Two-Photon Processes in Relativistic Heavy-Ion Colliders
}

\author{
J.-S. Wu, C. Bottcher, M. R. Strayer and C.M. Shakin' \\ Center for Computationally Intensive Physics, \\ Physics Division, Oak Ridge National Laboratory, Oak Ridge, TN 37831
}

Particle production via two-photon processes in relativistic heavy-ion colliders is discussed. We consider the coherent production, including its impact parameter dependence, and incoherent production. Particular attention is given to Higgs boson production in the energy regime of the LHC and the SSC.

\section{INTRODUCTION}

It was suggested several years ago that the highly peaked electromagnetic fields in ultra-relativistic heavy-ion collisions could be used as the sources to produce heavy particles coherently $[1,2]$. A variety of particle final states have been considered, including lepton pairs [3,4], W-pairs, Higgs bosons [5 - 8] and supersymmetric particles [9]. The advantage of production with heavy-ion beams is that the cross section of coherent production has $Z^{4}$ enhancement. At ligh energies the production is spread over a wide range of the impact parameter space of the colliding nuclei. The peripheral collisions can provide us with clean signals for detection.

Most of the calculations cited were carried out by using equivalent photon methods. However, in this approach the virtual photons are approximated by "on-shell" photons and the transverse momenta of the virtual photons are neglected. Therefore, the impact parameter dependence and transverse momentum spectra might be seriously wrong. Here is another approach which takes advantage of the rapidly developing high-performance computing resources. For the two-photon processes, production cross section can be written in terma of Feynman integrals in momentum space and integrated out numerically using Monte Carlo methods. It is straightforward to extend the approach to study the impact parameter dependence [8] and the incoherent production using structure functions [10].

We begin vrith the interaction Hamiltonian of quantum fields. The S-matrix can be defined as

$$
S=\mathrm{T} \exp \left\{-i \int d^{4} x \mathcal{H}_{\mathrm{int}}(x)\right\},
$$


where the operator $T$ gives the time-ordered product. The interaction Hamiltonian can be written as

$$
\mathcal{H}_{\text {int }}(x)=\mathcal{J}_{\mu}(x) A^{\mu}(x)+H_{\text {urt }}(x),
$$

where $\mathcal{J}_{1}$ is the electromagnetic source current of the colliding heavy ions and $H_{1, n}(x)$ is the interaction Hamiltonian of the quantum fields. The interaction Hamiltonians in the standard model for a varieiy of two photon processes are summarized in Table I. Since the search for the Higgs boson or the mechanism responsible for electroweak symmetry breaking is one of the primary goals of the next generation of supercolliders, we mainly concenirate on Higgs boson production in relativistic heavy-ion colliders.

The Higgs boson is a neutral scalar particle and does not directly couple to electromagnetic fields. However, the production of Higgs bosons via two virtual photons can occur through the production of charged particles and their subsequent fusion. The effective interaction between the Higgs boson and the electromagnetic fields is given by

$$
\mathcal{H}_{\text {int }}=\frac{e^{2}}{2} I: F^{\mu \nu} F_{\mu \nu} H:
$$

where $F_{\mu \nu}=\partial_{\mu} A_{\nu}-\partial_{\nu} A_{\mu}$.

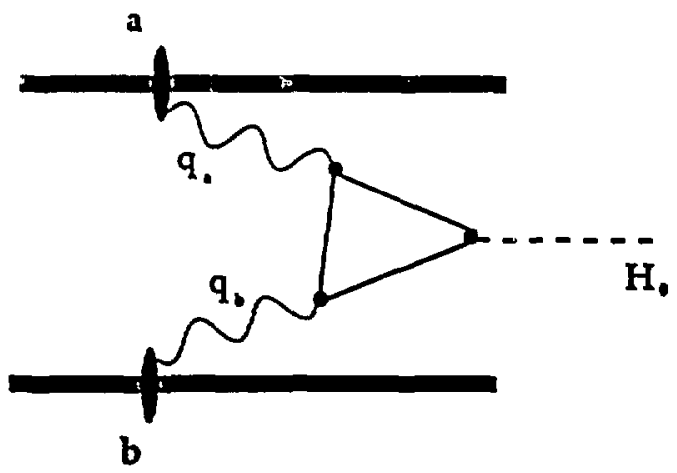

Fig. 1. The Feynman diagram for the effective coupling of the Higgs boson to two photons via a single loop. The loop sums over all combinations of charged fermions and bosons.

The lowest order Feynman diagram is shown in Fig. 1. In the figure the intermediate loops can be any charged particles, quarks, leptons or bosons. Their contributions can be written in terms of the above three-body vertex with an effective coupling $I$. In the standard model the effective coupling is given by

$$
I=\frac{g}{8 \pi^{2} M_{W}}\left(A_{\mathrm{q}}+A_{l}+A_{W}\right),
$$

and where each amplitude $A_{i}$ is only a function of the ratio of the masses, $\lambda_{i}=m_{i}^{2} / M_{H}^{2}$. Here, the contributions of the amplitudes come from the quarks, 
charged leptons, and $W^{ \pm}$, isons. Their explicit form can be found in refs.(11] and [12]. Generally, these amplitudes are sensitive to the mass ratio, and the major contribution is from the vector bosons and the top quark. For the case of an intermediate-mass Higgs boson of mass less than $200 \mathrm{GeV}$, and the top quark mass between the limits set by recent experiments, i.e. $130 \pm 30 \mathrm{GeV}$ [13], the effective coupling is not sensitive to the value of top quark mass. Thus, we choose $m_{t}=100 \mathrm{GeV}$ and the standard values for other quark masses in the discussion.

\section{COHERENT PRODUCTION}

For the coherent production we treat the electromagnetic fields as classical fields arising from the motion of two colliding nuclei. The potentials can be derived from Maxwell equations and written as

$$
A^{\mu}(x)=A_{a}^{\mu \prime}(x)+A_{b}^{\mu}(x),
$$

where $a$ and $\dot{o}$ label the nuclei and

$$
\begin{aligned}
A_{a, b}^{\prime \prime}(q) & =2 \pi Z_{a, b} \text { e } \delta\left(q^{0} \mp \beta q^{2}\right) \frac{F_{a, b}\left(-q^{2}\right)}{-q^{2}} \exp \left( \pm i \bar{q}_{\perp} \cdot \vec{b} / 2\right) u_{a, b}^{\prime \prime}, \\
u_{a, b}^{\prime \prime} & =(1,0,0, \pm \beta) .
\end{aligned}
$$

A few commento about the classical fields given in Eq. 6 are in order. $F_{a, b}\left(q^{2}\right)$ are the elastic form factors derived from the finite charge distribution of the nuclei. The velocity $u_{a, b}^{\mu}$ and the delta-function indicate that the fields can be obtained by boosting the nuclear Coulomb fields from the rest frame of the corresponding nucleus to the collider frame. The vector $b$ displaces the straight-line trajectories of the two colliding nuclei and will be identified as the impact parameter.

The Higgs production cross section is obtained from the S-matrix to the firat order in the Hamiltonian (3),

$$
\sigma_{a b-H_{0}}=\int \frac{d^{3} k}{(2 \pi)^{3} 2 \omega_{k}} \int d^{2} b|<\mathbf{k}| S^{(1)}|0>|^{2},
$$

where

$$
\begin{aligned}
\left.<\mathbf{k}\left|S^{(1)}\right| 0\right\rangle= & i e^{2} I \int \frac{d^{4} q_{1} d^{4} q_{2}}{(2 \pi)^{4}} \delta^{(4)}\left(q_{1}+q_{2}-k\right) \\
& \times\left[\left(q_{1} \cdot q_{2}\right) A_{\mu}\left(q_{1}\right) A^{\mu}\left(q_{2}\right)-q_{1 \mu} A^{\mu}\left(q_{2}\right) q_{2 \nu} A^{\nu}\left(q_{1}\right)\right] .
\end{aligned}
$$

When evaluating the S-matrix element, the classical fields are c-numbers rather than field operators, and can be taken out of the ordered product.

The integation over impact parameters can be carried out analytically, leading to the cross section expression

$$
\begin{aligned}
\sigma_{a b \rightarrow H_{0}}= & \frac{4 Z_{a}^{2} Z_{b}^{2}(4 \pi \alpha)^{4}|I|^{2}}{\beta^{2}} \int \frac{d^{3} k d^{2} q_{a \perp} d^{2} q_{b \perp}}{(2 \pi)^{5} 2 \omega_{k}} \delta^{(2)}\left(\vec{q}_{a \perp}+\vec{q}_{b \perp}-\vec{k}_{\perp}\right) \\
& \times \frac{F^{2}\left(-q_{a}^{2}\right)}{\left(q_{a}^{2}\right)^{2}} \frac{F^{2}\left(-q_{b}^{2}\right)}{\left(q_{b}^{2}\right)^{2}}\left[\left(q_{a} \cdot q_{b}\right)\left(u_{a} \cdot u_{b}\right)-\left(q_{n} \cdot u_{b}\right)\left(q_{b} \cdot u_{a}\right)\right]^{2} .
\end{aligned}
$$


The integral can be carried out numerically by the Monte Carlo method. Gauge invariance has been proved formally and tested numerically. This treatment also shows that both of the virtual photons $q_{a}, g_{b}$ are apace-like and considerably "offshell", since $-q_{a}^{2}=\left(\omega_{a} / \beta_{\gamma}\right)^{2}+\left(\vec{q}_{a \perp}\right)^{2}>0$ and $-q_{b}^{2}=\left(\omega_{b} / \beta \gamma\right)^{2}+\left(\vec{q}_{b \perp}\right)^{2}>0$.

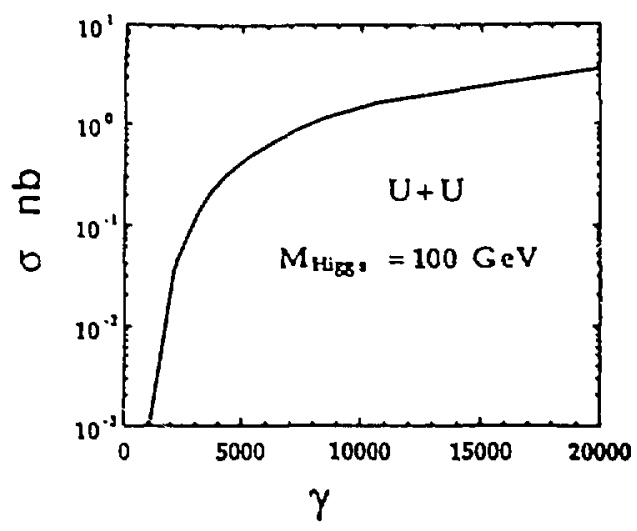

Fig. 2. The cross section for Higgs production in uranium collisions as a function of the Lorentz $\gamma$ of the beams. The top quaik mass is assumed to be $100 \mathrm{Gev}$.

Fig. 2 gives the cross section as a function of beam energy in uranium collisions for a Higgs mass $M_{H}=100 \mathrm{GeV}$. The qualitative behavior of the production cross section can be explained as follows. Consider the simple schematic model of a collision in which the nuclei each emit a virtual photon, $q_{a}^{\prime}, q_{b}^{\prime}$, in their respective rest frames, $q_{a}^{\prime}=-q_{b}^{\prime}=(0,0,0, q)$. The form factors $F\left(-q^{\prime 2}\right)^{2}$ represent the probabilities that each nucleus will emit the corresponding photon aud remain in their ground state. The form factors control the magnitude of the emission, which is strongly suppressed whenever the momentum transfer is much larger than the inverse nuclear size, i.e.,

$$
F\left(-q^{\prime 2}\right) \sim 0 \quad \forall-q^{\prime 2} \gg 1 / R .
$$

In the collision, these momenta are boosted with velocities $\pm \beta$, respectively,

$$
\begin{aligned}
& q_{a}^{\mu}=\gamma(\beta q, 0,0,+q), \\
& q_{b}^{\mu}=\gamma(\beta q, 0,0,-q) .
\end{aligned}
$$

The square of the total available energy from the photons, $S=4 \gamma^{2} \beta^{2} q^{2}$, is equal to the mass square of the produced Eiggs boson, i.e.

$$
M_{H}^{2}=4 \gamma^{2} \beta^{2} q^{2},
$$

while the "threshold" for the production,

$$
q \geq \frac{M_{u}}{2 \gamma \beta}
$$


For collisions at the LHC and the SSC the "thresholds" are about 14 and $6 \mathrm{MeV} / \mathrm{c}$, respectively, and are comparable to the inverse nuclear size for heavy nuclei, $R^{-1} \sim$ $30 \mathrm{MeV} / \mathrm{c}$. Thus, the coherent pair production can occur at these energies. For lower energies, the production is greatly suppressed by the form factor. As the energy is raised, the "threshold" climbs up the tail and the production cross section rises sharply. When the "threshold" reaches the plateau at small values of $Q^{2}$ the production dependence on energy saturates and goes over to its logarithmic asymptotic form. In the asymptotic regime the production is no longer suppressed by the form factor, and the magnitude of the cross section is not sensitive to the details of the form factor. It is only in this regime that the full $Z^{\prime \prime}$ coherent enhancement is recovered.

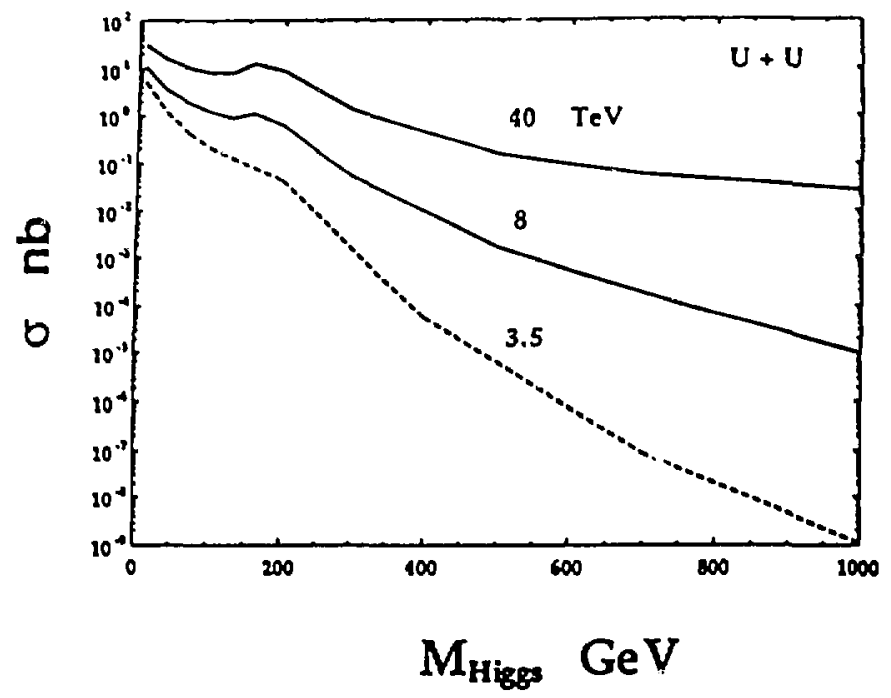

Fig. 3. The cross section for Higgs production in uranium collisions as a function of the Higgs mass at the LHC, the SSC, and the ELOISATRON. The top quark mass is assumed to be $100 \mathrm{GeV}$.

Fig. 3 gives the cross section in uranium collisions as a function of Higgs mass for the three beam energies $E=3.5,8.0$, and $40 \mathrm{GeV} / \mathrm{u}$, corresponding to the LHC, the SSC, and the proposed ELOISATRON, respectively. We take the charge-mass ratio as 0.4 in the expected beam energy per nucleon. The luminosity in heavy-ion colliders is typically about $1 \mathrm{pb} /$ year. We can estimate the Higgs boson production rates at these energies a few hundred to a few thousand per year for the Higgs mass $M_{H}=100 \mathrm{GeV}$. This figure also shows that at a large Higgs mess the production of the Higgs boson is very sensitive to the beam energies.

It can be shown from quantum field theory [10] that the above classical field approximation is especially good in the two-photon processes of heavy-ion collisions where the photon momentum transfer is small compared to the nuclear momenta. When the final nuclear momenta are approximated by their initial momenta in the expression for the production cross section, the classical field approximation can be recovered from the corresponding Feynman integral. As we shall discuss in the following section, the classical field approximation is particularly convenient for describing the dependence of particle production on the impact parameter. 


\section{HI. IMPAC'T PARAME'TER DEPENDENCE}

We are mostly interested in the clean signals provided by the peripheral col. lisons. In the central collisions the nuclei will undergo very strong excitations which remove them from the elastic flux. Therefore, we have to consider the im. pact parameter dependence of the production and exclude the production from central collisions.

As we have seen, the classical fields depend explicitly on the impact parameter. The S-matrix elements for the two-photon processes can be written as

$$
<f|S| 0>=\int \frac{d^{2} q_{\perp}}{(2 \pi)^{2}} M_{f}\left(\mathbf{q}_{\perp}\right) \exp \left\{i\left(\mathbf{q}_{\perp}-\mathbf{k}_{/ \perp} / 2\right) \cdot \mathbf{b}\right\},
$$

where $M_{f}$ is independent of $b$, and the delta-functions in the classical fields have been integrated out. The total cross section can be reduced to the following form

$$
\sigma_{a b \rightarrow H_{0}}=\int d^{2} b s(b) \sum_{f}|<f| S^{(1)}|0>|^{2},
$$

where $s(b)$ is a function excluding the flux from the central regime.

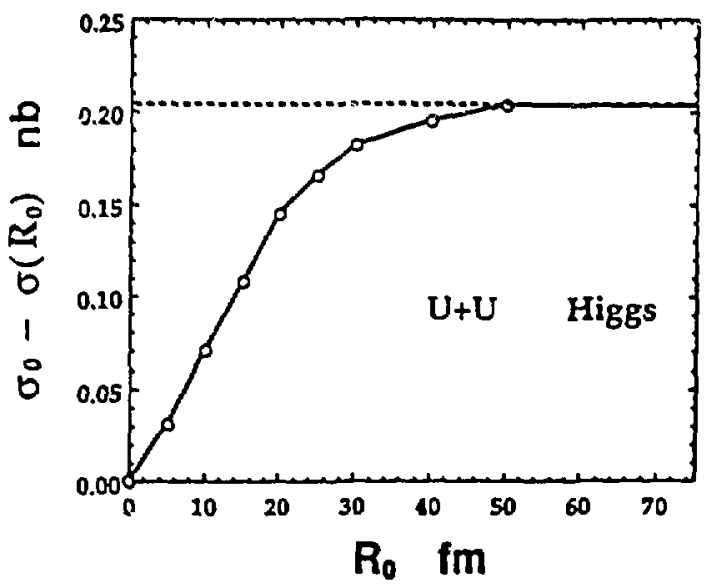

Fig. 4. The cross section $\sigma(0)-\sigma\left(R_{0}\right)$ for Higgs production in uranium collisions as a function of $R_{0}$, at beam energies per nucleon of $3.5 \mathrm{TeV}$. The top quark mass
is assumed to be $100 \mathrm{Gev}$.

With an explicit form of $s(b)$, we may carry out the integration over impact parameters and obtain

$$
\sigma_{a b \rightarrow f}=\sum_{f} \int \frac{d^{2} q_{1 \perp}}{(2 \pi)^{2}} \frac{d^{2} q_{2 \perp}}{(2 \pi)^{2}} M_{f}\left(\mathbf{q}_{1 \perp}\right) M_{f}\left(\mathbf{q}_{2 \perp}\right) S\left(q_{1 \perp}-q_{2 \perp}\right),
$$
where $S\left(q_{\perp}\right)=\int d^{2} b s(b) \exp \left\{i q_{\perp} \cdot b\right\}$. For convenience we may choose the simplest
assumption, i.e.

$$
s(b)=\theta\left(b-R_{1}\right)
$$


where $R_{n}$ is twice the nuclear radius, $2 R$. In Fig. 4 we plot the cross section $\sigma(0)-\sigma\left(R_{1}\right)$ for Higgs production as a function of the distance $R_{1}$ for collisions of uranium at a beam energy of $3.5 \mathrm{TeV}$ per nucleon.

From Fig. 4 we observe that a fairly large fraction of the cross section comes from impact parameters beyond peripheral collisions. Exclusion of central events at $R_{11}=2 R$, where $R$ is the nuclear radius, reduces the cross section for Higgs production by a factor of 1.9 at LHC (3.5 TeV), by a factor of 1.4 at SSC (8.0 $\mathrm{TeV}$ ), and by a factor of 1.2 at ELOISATRON ( $40 \mathrm{TeV})$.

\section{HIGGS DEC.AY}

For intermediate mass Higgs bosons, $M_{Z}<M_{H}<2 M_{11}$ and top quark masses $m_{l}>M_{n l} / 2,90$ percent of the Higgs decay width is into b-quark pairs, and the lifetime of the Higgs boson is about $10^{-2-1}$ seconds. Therefore, in experiments, we expect to observe the Higgs through its decay into hadron jets. This decay has been suggested by other authors $[5,7]$, and the corresponding crose section for the b-jet distribution is obtained by including the decay process $H_{0} \rightarrow b \bar{b}$, where

$$
\mathcal{H}_{\text {int }}=\left(\sqrt{2} G_{F}\right)^{1 / 2} m_{f} \bar{\psi} \psi_{f} H .
$$

The resulting cross section is

$$
\begin{aligned}
\sigma_{a b \rightarrow H_{0}-f j}= & \frac{4 Z_{a}^{2} Z_{b}^{2}(4 \pi \alpha)^{4}|I|^{2}}{\beta^{2}} \mathcal{C}_{f} \int \frac{d^{3} k_{f} d^{3} k_{f} d^{2} q_{a \perp} d^{2} q_{b \perp}}{(2 \pi)^{10} 2 E_{f} 2 E_{f}} \sum_{\rho, j, j}\left|\mathcal{U}\left(k_{f}, s_{f} ; k_{f}, s_{j}\right)\right|^{2} \\
& \times \delta^{(2)}\left(\vec{q}_{a \perp}+\vec{q}_{b \perp}-\vec{k}_{f \perp}-\vec{k}_{f \perp}\right) \frac{F^{2}\left(q_{a}^{2}\right)}{\left(q_{a}^{2}\right)^{2}} \frac{F^{2}\left(q_{b}^{2}\right)}{\left(q_{b}^{2}\right)^{2}} \\
& \times\left[\left(q_{a} \cdot q_{b}\right)\left(u_{a} \cdot u_{b}\right)-\left(q_{a} \cdot u_{b}\right)\left(q_{b} \cdot u_{a}\right)\right]^{2}
\end{aligned}
$$

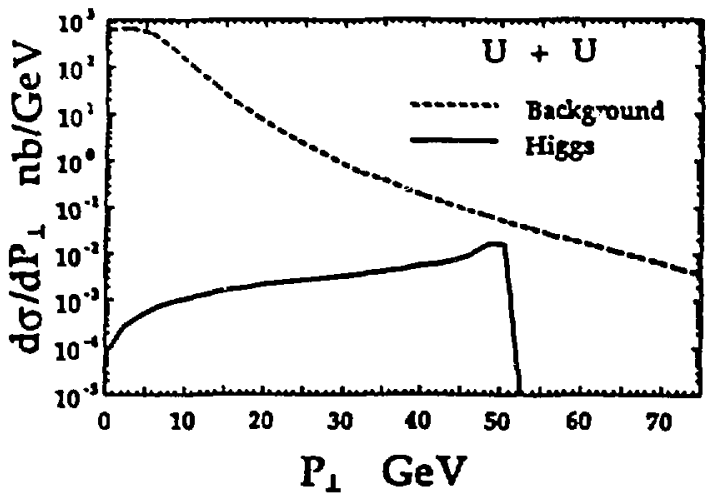

Fig.5. The transverse momentum distribution of the b-jets obtained from Higgs decay compared to the corresponding jet background obtained directly from twophoton production for uranium collisions at beam energies per nucleon of $3.5 \mathrm{TeV}$. The Higgs mass and the top quark mass are both assumed to be $100 \mathrm{GeV}$. 
The $H_{11} \rightarrow f \bar{f}$ vertex is given by

$$
u\left(k_{f}, s_{f} ; k_{f}, s_{f}\right)=\frac{\left(\sqrt{2} G_{F}\right)^{1 / 2} m_{f} \tilde{u}\left(k_{f}, s_{f}\right) v\left(k_{f}, s_{f}\right)}{\left(k_{f}+k_{f}\right)^{2}-M_{l l}^{2}+i \Gamma_{11} M_{l l}},
$$

while the colour degeneracies are $\mathcal{C}_{q}=3$ and $\mathcal{C}_{\ell}=1$, and $\Gamma_{H}$ is the total Higgs decay width. Note that the b-quark momentum in (19) is integrated out. From these equations we have computed the b-jet transverse momentum distribution arising from the Higgs decay. This is shown in Fig. 5 for uranium collisions at beam energies per nucleon of $3.5 \mathrm{TeV}$. Also shown in this figure is the corresponding background jet distribution obtained from the peripheral production of $\bar{b} \bar{b}$ pairs. We note that the background distributions are somewhat larger than those from the Higgs decay, indicating that intermediate mass Higgs bosons may be difficult to identify from the analysis of singles spectra.

One of the advantages of the Monte Carlo integration is that each Monte Carlo point can be treated as a weighted event. Any desired distribution may be accumulated by binning these events as the calculation proceeds. In general, more points are needed to calculate distributions than total cross sections.

\section{INCOIIERENT PRODUCTION}

By considering coupling constants, it is expected that the cross section of two-photon production of Higge bosons through inelastic events is smaller than gluon-gluon fusion by a factor of $\left(\alpha / \alpha_{0}\right)^{4}$, where $\alpha$ and $\alpha_{0}$ are the electromagnetic and strong coupling constants, respectively. However, it is worthwhile to make a quantitative comparison between the incoherent and coherent two-photon Higgs boson production using the direct Monte Carlo integration of the relevant Feynman diagrams.

For simplicity, we first consider the incoherent production in a p-p collision, in which the S-matrix can be factorized as the product of the proton current (inelastic) matrix elements and the two-photon production matrix elements. The production cross section is the incoherent sum of the inelastic processes, and summing over all possible final states yields an expression cast in terms of the proton structure function measured in the deep inelastic scattering.

The Higgs production cross section is obtained from the leading order term in the S-matrix, which is the third order in the Hamiltonian,

$$
\begin{aligned}
M= & (-i e)^{2}\left(-i e^{2} I\right) \frac{-i}{q_{a}^{2}} \frac{-i}{q_{b}^{2}}<X_{a}\left|\mathcal{J}_{\mu}\right| P_{a} s_{a}><X_{b}\left|\mathcal{J}_{\nu}\right| P_{b} s_{b}> \\
& \times\left(g_{a \lambda} g_{\sigma}^{\mu}-q_{a \sigma} g_{\lambda}^{\mu}\right)\left(q_{b}^{\lambda} g^{\nu \sigma}-q_{a}^{\sigma} g^{\nu \lambda}\right)(2 \pi)^{4} \delta^{4}\left(P_{\lambda_{a}}+P_{\lambda_{b}}+k-P_{a}-P_{b}\right) .
\end{aligned}
$$

In the collider frame the cross section can be written as

$$
\begin{aligned}
\sigma= & \frac{(4 \pi \alpha)^{4}|I|^{2}}{2 \beta} \int \frac{d^{3} k}{(2 \pi)^{3} 2 \omega_{k}} \frac{1}{2 \omega_{a}} \int \prod_{X_{a}} \frac{d^{3} p_{X_{a}}}{(2 \pi)^{3} 2 \omega_{X_{a}}} \frac{1}{2 \omega_{b}} \int \prod_{X_{b}} \frac{d^{3} p_{X_{S_{b}}}}{(2 \pi)^{3} 2 \omega_{X_{b}}} \\
& \times \frac{1}{2} \sum_{a_{a}} \sum_{x_{X_{a}}}<X_{a}\left|\mathcal{J}_{\mu}\right| P_{a} s_{a}><P_{a} s_{a}\left|\mathcal{J}_{\mu^{\prime}}\right| X_{a}>\left(\frac{1}{q_{a}^{3}}\right)^{2}
\end{aligned}
$$




$$
\begin{aligned}
& \frac{1}{2} \sum_{a b} \sum_{x_{b}}\left\langle x_{b}\left|\mathcal{J}_{1}\right| P_{b}, s_{b}><P_{b} s_{b}\left|\mathcal{J}_{1}\right| X_{b}>\left(\frac{1}{q_{b}^{2}}\right)^{2}\right. \\
& \left.\left.\times 4\left\{\left(q_{a} \cdot q_{b}\right) g^{\prime \prime \prime \prime}-q_{a}^{\prime \prime} q_{b}^{\prime \prime}\right)\right\}\left\{\left(q_{a} \cdot q_{b}\right) g^{\prime \prime u^{\prime \prime}}-q_{a}^{\prime \prime} q_{b}^{\prime \prime \prime}\right)\right\}(2 \pi)^{\prime} \delta^{\prime \prime}\left(q_{a}+q_{b}-k\right),
\end{aligned}
$$

where the sums are over all the possible hadronic final states $X_{a}$ and $X_{i}$, and $\beta$ is the velocity of the proton in the collider frame. We may introduce a structure function defined by

$$
\begin{aligned}
& W_{M \mu^{\prime}}\left(x, Q^{2}\right)=\frac{1}{4 M_{n}} \int \prod_{.1} \frac{d^{3} p_{. X}}{(2 \pi)^{3} 2 \omega_{. x}} \frac{1}{2} \sum_{s} \sum_{\theta x}\left\langle X\left|\mathcal{J}_{\mu}\right| P s\right\rangle \\
& x<P s\left|\mathcal{J}_{\mu^{\prime}}\right| X>(2 \pi)^{3} \delta^{-1}\left(P_{. X}-P+q\right),
\end{aligned}
$$

where $Q^{2}=-q^{2}$ and $x=q^{2} / 2 M_{n} \nu$ and $M_{n}$ is the proton mass. Here $\nu$ is the time component of the momentum transfer $q$ in the proton rest frame. It can be proven that the proton electromagnetic structure function has a general form

$$
\begin{aligned}
W_{\mu \nu}\left(x, Q^{2}\right)= & W_{1}\left(x, Q^{2}\right)\left(-g_{\mu \nu}+\frac{q_{\mu} q_{\nu}}{q^{2}}\right) \\
& +\frac{1}{M_{n}^{2}} W_{2}\left(x, Q^{2}\right)\left(P_{n}-\frac{1}{2 x} q_{\mu n}\right)\left(P_{\nu}-\frac{1}{2 x} q_{\nu}\right) .
\end{aligned}
$$

It is usual to identify the two structure functions

$$
M_{n} W_{1}\left(x, Q^{2}\right)=F_{1}\left(x, Q^{2}\right), \quad|\nu| W_{2}\left(x, Q^{2}\right)=F_{2}\left(x, Q^{2}\right),
$$

which satisfies the Callen-Gross relation in the scaling limit.

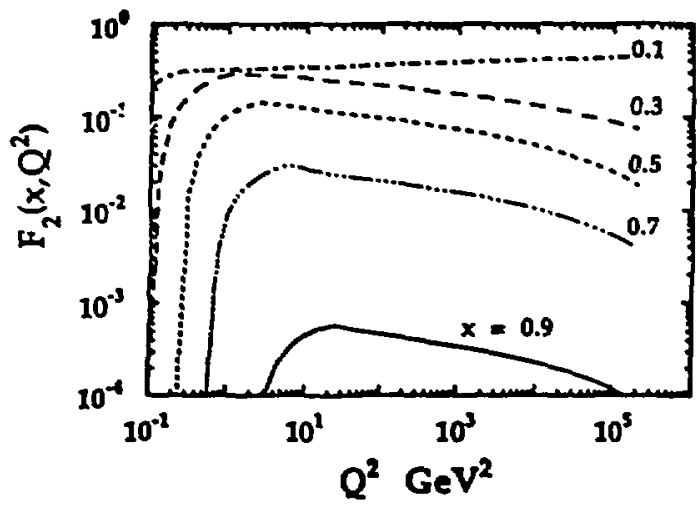

Fig. 6. The proton structure function $F_{2}$ as a function of $x$ and $Q^{2}$.

Therefore, the total cross section is given by

$$
\begin{aligned}
\sigma= & \frac{(4 \pi \alpha)^{4}|I|^{2}}{2 \beta \gamma^{2}} \int \frac{d^{3} k}{(2 \pi)^{3} 2 \omega_{k}} \int \frac{d^{4} q_{a}}{(2 \pi)^{2}} W_{\mu \mu^{\prime}}^{(a)}\left(x_{a}, Q_{a}^{2}\right) W_{{ }^{\prime} \nu^{\prime}}^{(b)}\left(x_{b}, \dot{Q}_{b}^{2}\right) \\
& \left.\left.\times\left(\frac{1}{q_{a}^{2}}\right)^{2}\left(\frac{1}{q_{b}^{2}}\right)^{2} 4\left\{\left(q_{a} \cdot g_{b}\right) g^{\mu \nu}-g_{a}^{\nu} q_{b}^{\prime \prime}\right)\right\}\left\{\left(g_{n} \cdot q_{b}\right) g^{\prime \prime^{\prime \prime} \nu^{\prime}}-q_{n}^{\prime \prime} q_{b}^{\prime \prime \prime}\right)\right\} .
\end{aligned}
$$


For heavy-ion collisions we may replace the proton structure function by the nuclear structure function, which is, as a first approximation neglecting the nuclear EMC effect, the incoherent sum of the structure functions for all nucleons, i.e.

$$
W_{i}\left(x, Q^{2}\right)=Z W_{1}^{(p)}\left(x, Q^{2}\right)+N W_{i}^{(n)}\left(x, Q^{2}\right)
$$

where $i=1,2$, and $Z$ and $N$ are the charge number and the neutron number, respectively. The neutron structure function is taken to be

$$
F_{2}^{(n)}\left(x, Q^{2}\right)=(1-x) F_{2}^{(p)}\left(x, Q^{2}\right)
$$

which is a good approximation except for large values of $x$, which do not contribute much to the total cross section. In practice, the structure function is fitted to the experimental data in a limited kinematic range and extrapolited to the whole phase space consistent with energy-momentum conservation. The conservation is expressed by the delta-function appropriate to scattering from a composite system. With the inclusion of the higher-twist coefficient and target mass corrections given in ref. [14], the proton structure function for the leading twist is fitted to the EMC data [15]. Since the inelastic scattering of nucleon has a threshold energy of $t_{0}=\left(M_{n}+m \pi\right) c^{2}$, where $m_{\pi}$ is the pion mass, the structure functions are taken to be zero below the threshold. For numerical convenience, an interpolation is introduced between $t_{0}$ and $3 t_{0}$. Fig. 6 shows the variation of the proton structure function with $x$ and $Q^{2}$.

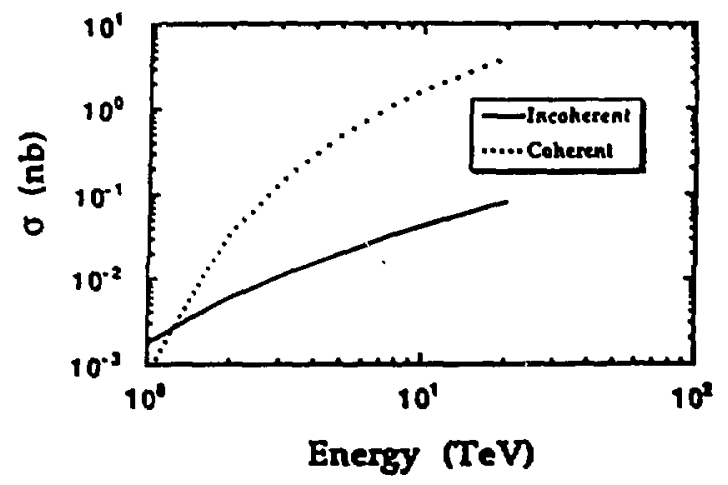

Fig. 7. The crose section for the incoherent Higgs production as a function of the beam energy per nucleon for uranium colisions. The Higgs mass and the top quark mase are both assumed to be $100 \mathrm{GeV}$.

The total cross section for Higgs production in the heavy-ion collider for uranium collisions is shown in Fig. 7 as a function of energy. The results are compared with the coherent production. The coherent production is seen to have a sharp rise in the energy from 1 to $2 \mathrm{TeV}$ per nucleon, reflecting the suppression of the cross section by the nuclear form factor at lower beam energies. At higher bombarding energies, the coherent production dominates the incoherent. The ratio scales roughly as $Z^{4} / A^{2}$. 


\section{CONCIJUSION}

We have calculated the colierent and incoherent production of Higgs bosons in ultrarelativistic heavy-ion collisions from the standard model. We use perturbation theory to calculate the two-photon processes and carry out the Feynman integrals numerically by Monte Carlo methods. For coherent production we use a classical field approximation to the nuclear electromagnetic fields. This method is a good approximation for coherent processes involving heavy ions, and it is particulacly suited for the description in impact parameter space when the classical irajectories of heavy ions are experimentally tagged. The results show that beyond some critical energy the coherent production with $Z^{4}$ enhancement is significant and dominates the incolierent; asymptotically, the production cross section increases with energy as $(\ln \gamma)^{3}$. However, the production is very sensitive to the beam energy below the critical energy. Since the coherent production provides clean signatures in peripheral collisions, the prospect of using heavy ions as a means of testing the standard model and searching for the Higgs boson is excellent at the higher energy machines.

\section{ACKNOWLEDGMENTS}

This research was sponsored by the Division of Chemical Sciences, Office of Basic Energy Sciences, and by the Division of Nuclear Physics of the U.S. Department of Energy under contract No. DE-AC05-84OR21400 with Martin Marietta Energy Systems Inc, and by the National Science Foundation and the Faculty Research Award Program of the City Jniversity of New York.

-Permanent address: Department of Physics, and Center for Nuclear Theory, Brooklyn College of the City University of New York, Brooklyn, New York 11210.

TABLE I. The table of interaction Hamiltonian used in the particle production of relativistic heavy-ion colliders, where : : indicates the normal ordered product.

"In the standard model $\mathcal{X}=1$.

\begin{tabular}{|c|c|}
\hline with photons & $H_{\text {int }}(x)$ \\
\hline charged fermions & $e: \psi \gamma^{\mu} \psi A_{\mu}:$ \\
\hline$W^{ \pm}$bosons & $\begin{aligned} &-i e:\left\{\mathcal{K}\left(\partial_{\mu} A_{\nu}-\partial_{\nu} A_{\mu}\right) W^{-\mu} W^{+\nu}\right. \\
&+\left(\partial_{\mu} W_{t}^{+}-\partial_{\nu} W_{\mu}^{+}\right) A^{\mu} W^{-\nu} \\
&\left.+\left(\partial_{\mu} W_{\nu}^{-}-\partial_{\nu} W_{\mu}^{-}\right) W^{+\mu} A^{\nu}\right\}: \\
&+e^{2}:\left(W_{\mu}^{+} W^{-\mu} A_{\nu} A^{\nu}-W_{\mu}^{+} W_{\nu}^{-} A^{\mu} A^{\nu}\right):\end{aligned}$ \\
\hline charged scalar bozons & $\begin{array}{c}-i e: A_{\mu}\left(\phi^{\dagger} \theta^{\mu} \phi-\partial^{\mu} \phi^{\dagger} \phi\right) \\
-e^{2}: A_{\mu} A^{\mu} \phi^{\dagger} \phi:\end{array}$ \\
\hline neutral scalar bosons & $\frac{c^{2}}{2} I: F_{m, F^{\prime \prime \prime}} \phi:$ \\
\hline \multicolumn{2}{|l|}{ with the Higgs boson } \\
\hline fermions & $\left(\sqrt{2} G_{F}\right)^{1 / 2} m_{f}: H \bar{f} f:$ \\
\hline weak bosons & $\left(\sqrt{2} G_{F}\right)^{1 / 2}:\left(2 M_{\|}^{2} \cdot W_{\| \prime}^{+} W^{-\mu}+M_{Z}^{2} Z_{\mu l} Z^{\prime \prime}\right) H:$ \\
\hline
\end{tabular}




\section{REFERENCES}

'H. Gould, in Prac. Atomic Theory Workshop on Relativistic and QED Effects in Heavy Atoms, AIP Conf. Proc., Vol. 136 (AIP, New York, 1985) p. 66 .

2 C. BotTcher and M.R. STrayer, in "Physics of Strong Fields", edited by W. Greiner, (Plenum, New York, 1987), Vol. 153, page 629; "Proceedings of the Second Workshop on Experiments and Detectors for the RHIC", Berkeley, California, May 25-29, 1987, page 279, (LBL-24604); Nucl. Inst. and Meth.

' C. BotTcher and M.R. Strayer, Phys. Rev. D39, 1330 (1989).

+ G. Baur and C.A. Bertulani, Phys. Rep. 161, 299 (1988).

${ }^{5}$ M. Grabiak, B. Müller, W. Greiner, G. Soff, and P. Koch, J. Phys. G15, L25 (1989).

${ }^{6}$ Elena Papageorgiu, Phys. Rev. D40, 92 (1989); Nucl. Phys. A498, 593c (1989).

7 M. Drees, J. Ellis ANd D. Zeppenfeld, Phy's. Lett. 223B, 454 (1989).

' C. Bottcher, A.K. Kerman, M.R. Strayer and J.S. Wu, Particle World, Vol. 1, No. 6, 174 (1990).

9 G. Soff, J. Rau, M. Grabiak, B. Müller and W.Greiner, Production of super-symmetric particles and Higgs bosons in ultrarelativistic heavyion collisions, Preprint, 1989, (GSI-89-55).

I" J.S. Wu, C. BotTcher, M.R. Strayer and C.M. Shakin, Phys. Rev. C43 2422 (1991).

$"$ J.F. Gunion, H.E. Haben, G.L. Kane, and S. Dawson, "The Higgs Hunter's Guide", (Addison Wesley, New York, 1990).

12 J.S. Wu, C. Bottcher, M.R. Strayer and A.K. Kerman, Ann. Phys. (in Press, 1991).

13 J. Ellis ANd G.L. Fogli, Phys. Lett. B249, 543 (1990).

14 A. Milsztajn, Saclay Report No. DPHPE90-07, (1990).

15 J.J. AuberT et. al., The European Muon Collaboration, Nucl. Phys., B25日, 189 (1985). 